\title{
Associations and Outcomes of Septic Pulmonary Embolism
}

\author{
Umesh Goswami ${ }^{*}$, , Jorge A. Brenes ${ }^{2}$, Gopal V. Punjabi ${ }^{3}$, Michele M. LeClaire ${ }^{4}$ \\ and David N. Williams ${ }^{5}$
}

\author{
${ }^{I}$ Department of Pulmonary, Allergy, Critical Care and Sleep Medicine, University of Minnesota, Minneapolis, MN, USA \\ ${ }^{2}$ Department of Medicine, Division of Cardiovascular Diseases, Mayo Clinic, Rochester, MN, USA \\ ${ }^{3}$ Department of Radiology, Hennepin County Medical Center, Minneapolis, MN, USA \\ ${ }^{4}$ Division of Pulmonary and Critical Care Medicine, Department of Medicine, Hennepin County Medical Center, \\ Minneapolis, MN, USA \\ ${ }^{5}$ Division of Infectious Diseases, Department of Medicine, Hennepin County Medical Center and University of \\ Minnesota, Minneapolis, MN, USA
}

\begin{abstract}
Background: Septic pulmonary embolism is a serious but uncommon syndrome posing diagnostic challenges because of its broad range of clinical presentation and etiologies.

Objective: To understand the clinical and radiographic associations of septic pulmonary embolism in patients presenting to an acute care safety net hospital.

Methods: We conducted a retrospective analysis of imaging and electronic health records of all patients diagnosed with septic pulmonary embolism in our hospital between January 2000 and January 2013.

Key Results: 41 episodes of septic pulmonary embolism were identified in 40 patients aged 17 to 71 years (median 46); 29 (72\%) were men. Presenting symptoms included: febrile illness (85\%); pulmonary complaints (66\%) including pleuritic chest pain (22\%), cough (19\%) and dyspnea (15\%); and those related to the peripheral foci of infection (24\%) and shock (19\%). Sources of infection included: skin and soft tissue (44\%); infective endocarditis (27\%); and infected peripheral deep venous thrombosis (17\%). 35/41 (85\%) were bacteremic with staphylococcus aureus. All patients had peripheral nodular lesions on chest CT scan. Treatment included intravenous antibiotics in all patients. Twenty six (63\%) patients required pleural drainage and/or drainage of peripheral abscesses. Seven (17\%) patients received systemic anticoagulants. Eight (20\%) patients died due to various complications.

Conclusion: The epidemiology of septic pulmonary embolism has broadened over the past decade with an increase in identified extrapulmonary, non-cardiac sources. In the context of an extrapulmonary infection, clinical features of persistent fever, bacteremia and pulmonary complaints should raise suspicion for this syndrome, and typical findings on the chest CT scans confirm the diagnosis. Antibiotics, local drainage procedures and increasingly, anticoagulation are keys to successful outcomes.
\end{abstract}

Keywords: Endocarditis, Lemierre's syndrome, lung infection, pulmonary embolism, septic thrombophlebitis, Staphylococcus.

\section{INTRODUCTION}

Septic pulmonary embolism (SPE) is an uncommon syndrome characterized by embolization of infected thrombi from a primary infectious site into the venous circulation with implantation into pulmonary vasculature resulting in parenchymal infection. The clinical presentation varies from an insidious illness with fever and respiratory symptoms such as cough, shortness of breath, and pleuritic chest pain to an acute sepsis syndrome. In the right clinical context, the

*Address correspondence to this author at the Department of Pulmonary, Allergy, Critical Care and Sleep Medicine, University of Minnesota, 420 Delaware St. SE, Minneapolis, MN 55455, USA; Tel: 612-646-1146;

E-mail: goswa009@umn.edu diagnosis of SPE is often triggered by a characteristic pattern seen on chest imaging studies with multiple, bilateral peripheral pulmonary nodules often with cavitations.

Historically, SPE was most commonly associated with Lemierre's syndrome [1], post-partum septic pelvic thrombophlebitis $[2,3]$ and right-sided infective endocarditis in IV drug users [1]. Within the last decade, attention has been drawn to changes in the epidemiology, including the importance of infected vascular catheters and implantable devices [4] and more recently septic thrombophlebitis due to contiguous deep soft tissue or bone infection of the extremities [5]. Thus an increasing number of patients present with symptoms related to an extrapulmonary source of infection, different from the classic presentation reported in the older literature [1]. 
In order to better understand the evolving etiology of SPE we undertook a retrospective analysis of all cases seen at our institution over the past 13 years.

\section{MATERIALS AND METHODS}

The study was conducted at the Hennepin County Medical Center, a 360 bed Level I trauma center and an urban teaching hospital in Minneapolis, Minnesota. After receiving permission from the hospital's office of human subjects research, a computer aided search of the electronic medical record and the imaging database was performed to identify cases of SPE between January 2000 and January 2013. The terms SPE and its variations were utilized for the purpose of the database search. The records were reviewed for each identified patient and the diagnosis of SPE confirmed if the subject met the following pre-specified case definition as described elsewhere in literature $[4,5]$ : characteristic radiologic findings on CT imaging; a clinical illness with systemic infection and concurrent bacteremia; and an identified extrapulmonary source of infection. Resolution of typical radiological lesions with appropriate antibiotic treatment further helped strengthen the diagnosis.

All images were reviewed by a radiologist (GVP) and all patients' electronic medical records were reviewed independently by 2 of the authors (UG and JAB) with further review by a third author (DNW) in questionable cases. Demographic variables obtained included age at diagnosis and gender. Clinical data included: relevant comorbidities, clinical presentation, microbiologic data, cardiac and other imaging, treatment modalities including surgical procedures, duration of hospitalization, duration and type of antibiotic therapy and treatment outcomes.

\section{RESULTS}

The study population consisted of 40 patients aged 17-71 years (median age 46 years). There were 29 men and 11 women (Table 1). One patient had two episodes of SPE, one year apart. In 26 of the 41 cases $(63 \%)$ radiographic features of SPE were detected within 72 hours of presentation.

Presenting symptoms included a febrile illness (85\%), pulmonary symptoms, most typically pleuritic chest pain $(22 \%)$, cough $(19 \%)$ and dyspnea (15\%). Eight patients $(19 \%)$ presented with septic shock. Thirteen patients (32\%) were admitted directly to the medical intensive care unit because of the acuity of their illness, $8(61 \%)$ of whom did not survive the hospitalization (Table 2). Identified comorbidities at the time of admission included IV drug abuse (IVDA) (15/41), endstage renal disease $(10 / 41)$, diabetes mellitus (7/41) and immunosuppressive states (3/41). A potential source of infection was identified in all but one patient. These included skin and soft tissue sites (cellulitis 1 case, infected skin ulcers 4 cases, deep subcutaneous abscess 13 cases) predominantly; followed by infective endocarditis, indwelling IV devices, and peripherally infected deep venous thrombosis (Fig. 1A). Microbiologic studies underscored the importance of staphylococcus aureus as the predominant cause (35 cases, 21 due to Methicillin Sensitive Staphylococcus aureus- MSSA and 14 Methicillin Resistant - MRSA) (Fig. 1B). Thirty seven patients had positive blood cultures (Fig. 1C).
Table 1. Baseline characteristics of $40^{\#}$ patients diagnosed and treated for SPE between years 2000 and 2013.

\begin{tabular}{|c|c|}
\hline Characteristics & No. $(\%)$ \\
\hline Age - median (range) years & $46(17-71)$ \\
\hline Males & $29(72)$ \\
\hline Females & $11(28)$ \\
\hline \multicolumn{2}{|l|}{ Relevant co-morbidities* } \\
\hline IV drug use & $15(36)$ \\
\hline End stage renal disease with ongoing dialysis & $10(24)$ \\
\hline Chronic liver disease (cirrhosis, hepatitis C) & $8(19)$ \\
\hline Diabetes mellitus & $7(17)$ \\
\hline Alcoholism and non IV drug use & $4(10)$ \\
\hline Polytrauma & $3(7)$ \\
\hline Congestive heart failure & $2(5)$ \\
\hline Neurological disorders (seizures, stroke, dementia) & $2(5)$ \\
\hline \multicolumn{2}{|l|}{ Immunosuppressive states } \\
\hline Post solid organ transplant & $2(5)$ \\
\hline HIV/AIDS & $1(2)$ \\
\hline Postpartum & $1(2)$ \\
\hline None & $1(2)$ \\
\hline \multicolumn{2}{|l|}{ Presenting signs/symptoms* } \\
\hline Febrile illness & $35(85)$ \\
\hline \multicolumn{2}{|l|}{ Respiratory } \\
\hline Chest pain & $9(22)$ \\
\hline Cough & $8(19)$ \\
\hline Dyspnea & $6(15)$ \\
\hline Hemoptysis & $4(10)$ \\
\hline Hypotension/shock & $8(19)$ \\
\hline Neurological (altered mentation, seizure, syncope) & $6(15)$ \\
\hline Musculoskeletal pain & $8(19)$ \\
\hline Sore throat & $2(5)$ \\
\hline
\end{tabular}

40 patients with 41 episodes of SPE (1 patient had 2 separate SPE episodes).

*Not absolute percentages as multiple patients had more than one co-morbidities and symptoms.

All patients had echocardiograms undertaken, with the exception of one who died within one day of admission. 18 patients $(45 \%)$ underwent transesophageal echocardiograms. Of the 11 cases of infective endocarditis (IE), 8 involved the tricuspid, 2 the mitral and one the pulmonic valve. 10 of these patients had definite IE according to the modified LiDuke criteria [6].

All patients, as required by definition, had chest CT imaging and the majority (36/41) also had chest radiographs performed. All had peripheral nodular lesions on the CT, with cavitations in 29/41 (71\%) (Table 3). About half (54\%) of the patients also had non-nodular infiltrates including some (4/41) with ground glass infiltrates. Twenty one 
A. Foci of primary infection

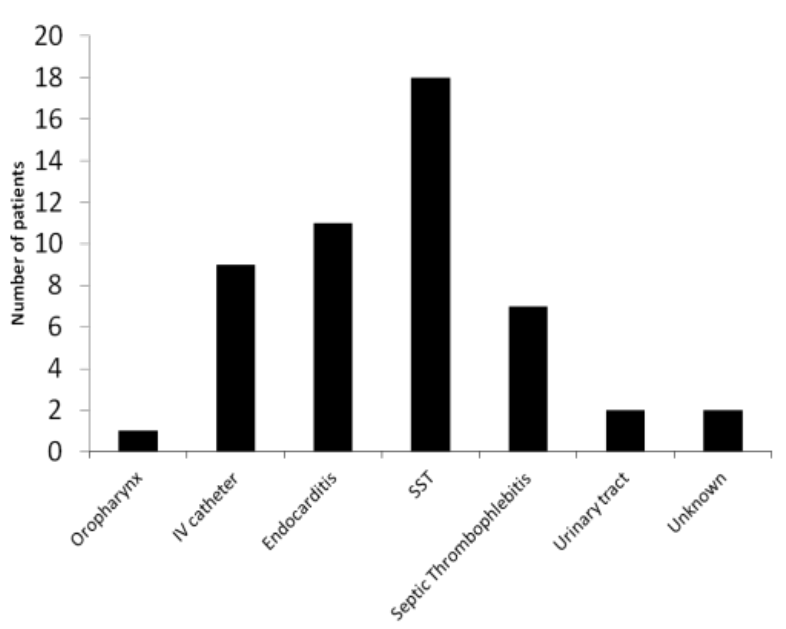

B. Pathogens

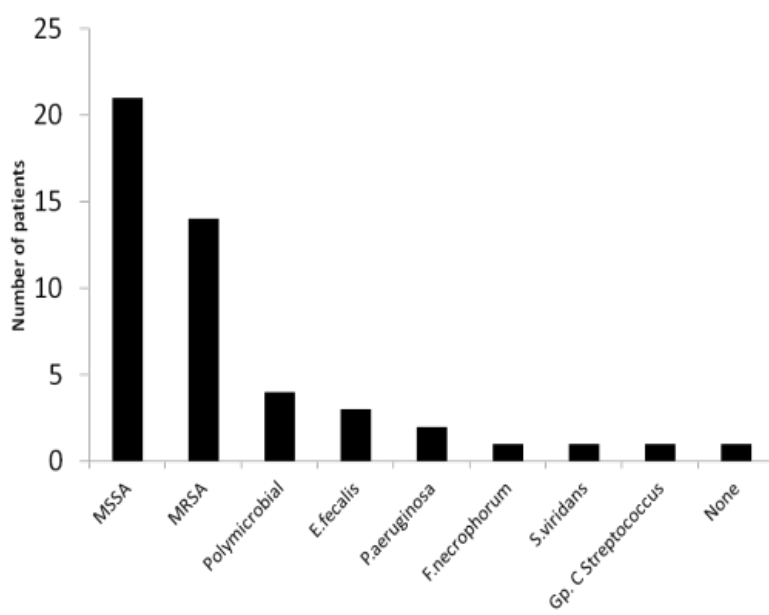

C. Sources of positive identification

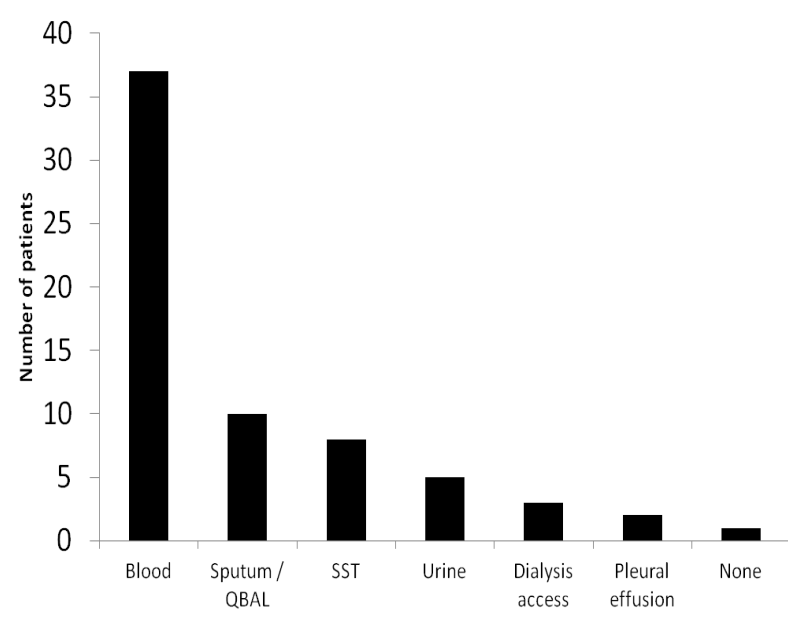

Fig. (1). Microbiology and culture characteristics in 41 cases (40 patients) with SPE.

patients (51\%) had pleural effusions noted on imaging. All patients received antibiotics guided by results of cultures for a median duration of 6 weeks (range: 3 to 12 weeks). Twelve patients $(29 \%)$ required a pulmonary procedure, most
Table 2. Management and outcome variables.

\begin{tabular}{|c|c|}
\hline Characteristics & $\begin{array}{l}\text { No./Total No. (\%) } \\
\text { * Median (Range) } \\
\text { Weeks }\end{array}$ \\
\hline \multicolumn{2}{|l|}{ Primary admission bed type } \\
\hline $\mathrm{ICU}$ & $13 / 41(32)$ \\
\hline Telemetry & $13 / 41(32)$ \\
\hline General floor & $15 / 41(36)$ \\
\hline Antibiotic use & $41 / 41(100)$ \\
\hline Duration* & $6(3-12)$ \\
\hline Systemic anticoagulant use & $9 / 15(60)$ \\
\hline \multicolumn{2}{|l|}{ Outcome } \\
\hline Discharged with improvement & $33 / 41(80)$ \\
\hline Death & $8 / 41(20)$ \\
\hline \multicolumn{2}{|l|}{ Causes of mortality } \\
\hline Cardiovascular (refractory shock) & $4 / 8(50)$ \\
\hline $\begin{array}{l}\text { Respiratory (respiratory failure, } \\
\text { massive hemoptysis, } \\
\text { pulmonary hemorrhage) }\end{array}$ & $3 / 8(38)$ \\
\hline Other (failure to thrive) & $1 / 8(12)$ \\
\hline
\end{tabular}

Table 3. CT imaging characteristics and findings.

\begin{tabular}{|c|c|}
\hline Characteristics & No. (\%) of Patients \\
\hline \multicolumn{2}{|l|}{ Technique } \\
\hline Without IV contrast & $12(29)$ \\
\hline With IV contrast & $29(71)$ \\
\hline \multicolumn{2}{|l|}{ Location of lesions } \\
\hline Central & $4(10)$ \\
\hline Peripheral & $41(100)$ \\
\hline Diffuse & $2(5)$ \\
\hline \multicolumn{2}{|l|}{ Types of lesions } \\
\hline Nodular & $41(100)$ \\
\hline Non-nodular & $22(54)$ \\
\hline Ground glass & $4(10)$ \\
\hline Cavitory & $29(71)$ \\
\hline Pleural effusion & $21(51)$ \\
\hline \multicolumn{2}{|l|}{ Number of lesions } \\
\hline Solitary & $3(7)$ \\
\hline $2-5$ & $18(45)$ \\
\hline $6-10$ & $5(12)$ \\
\hline$>10$ & $15(36)$ \\
\hline \multicolumn{2}{|l|}{ Frequency of cavitations } \\
\hline None & $12(29)$ \\
\hline Some & $11(27)$ \\
\hline All & $18(44)$ \\
\hline
\end{tabular}


typically thoracentesis or chest tube placement. All of the drained effusions were characterized as exudative. Ten patients $(24 \%)$ required source control with local incision and drainage of a peripheral site of infection or IV access removal (2 patients)/revision. Seven patients $(17 \%)$ required systemic anticoagulation for identified thromboses. Thirty three patients $(80 \%)$ improved and were discharged from the hospital. Twenty nine patients had follow up imaging documenting complete resolution of the lesions following antibiotic treatment.

\section{DISCUSSION}

SPE is an uncommon and serious disorder associated with significant morbidity and mortality. It remains a difficult diagnosis in part because of its varied etiology and clinical presentations. In this study we highlight the broad spectrum of etiologic causes that have changed over time. In one of the largest early studies reported by MacMillan et al. [1], $75 \%$ of cases of SPE were seen in the setting of rightsided infective endocarditis secondary to IV drug abuse, some with incidental superficial skin abscesses. More recently, Cook et al. [4] reported the role of infected IV catheters and devices as a source of SPE. The current series draws attention to the importance of skin, deep soft tissue and bone infections of the extremities as a key source. Infections with contiguous septic thrombophlebitis leading to SPE have been well established in pediatric populations [3] but have only recently been emphasized in adults by Brenes et al. [5]. Irrespective of the initial site of infection, there is a common sequence of events that triggers this syndrome (Fig. 2). An extrapulmonary site of infection can allow extravasation or translocation [7] of an organism, most commonly bacterial, into the systemic venous circulation. Once in the bloodstream, the pathogen can produce damage directly through toxins [8] and indirectly via inflammatory mediators [9], which may occasionally promote local thrombosis [10], which serves as an additional nidus for proliferation of the bacteria. Embolization of these thrombi into the pulmonary circulation allows for metastatic parenchymal infection of the lungs, even in the absence of cardiac valvular involvement. Special attention should be given to Staphylococcus aureus, which can provoke an intense inflammatory reaction, with direct endothelial damage via cytotoxins (e.g. Panton-Valentine leukocidin) and enzymatic mechanisms (e.g. Coagulase), culminating in septic pulmonary emboli, with concomitant septic thrombophlebitis in a minority of the cases [5].

The diagnosis of SPE should be suspected in a febrile bacteremic patient, with an identified extrapulmonary source of infection who develops secondary pulmonary symptoms such as pleuritic chest pain, dyspnea and cough. Although plain chest radiographs are usually obtained in such patients, they are notorious for their low sensitivity [11] with up to $22 \%$ being interpreted as normal in our study. However, if the suspicion is high, chest CT scans should be obtained to further evaluate the respiratory symptoms. The diagnosis of septic pulmonary embolism is strongly supported by characteristic findings including multiple bilateral peripheral nodules, often pleural based, with or without cavitary changes $[11,12]$. These chest CT findings typically lead to cardiac imaging because of the association with right-sided infective endocarditis. The absence of obvious cardiac valvular lesions should prompt careful consideration of other potential sources of infection including IV and other devices as well as thrombophlebitis due to skin and soft tissue infections. Additional imaging including ultrasound and directed CT scanning studies greatly aid in delineating the root cause of infection. Such work-ups may also help differentiate other syndromes with similar imaging characteristics and guide therapy [13].

The identification of a microbiologic etiology with blood, sputum and pleural fluid cultures and when relevant, deep soft tissue, bone and explanted device cultures [14] is critical. The microbiologic diagnosis may be hampered by early initiation of empiric antibiotic therapy [15] and this was the likely explanation of our inability to identify the primary source of infection in one patient.

Staphylococcus aureus and MSSA in particular, remains the most likely pathogen as evidenced in our series. This is consistent with the higher frequency of skin and soft tissue infections as the initial extrapulmonary source. The microbiology of SPE may vary due to a number of factors

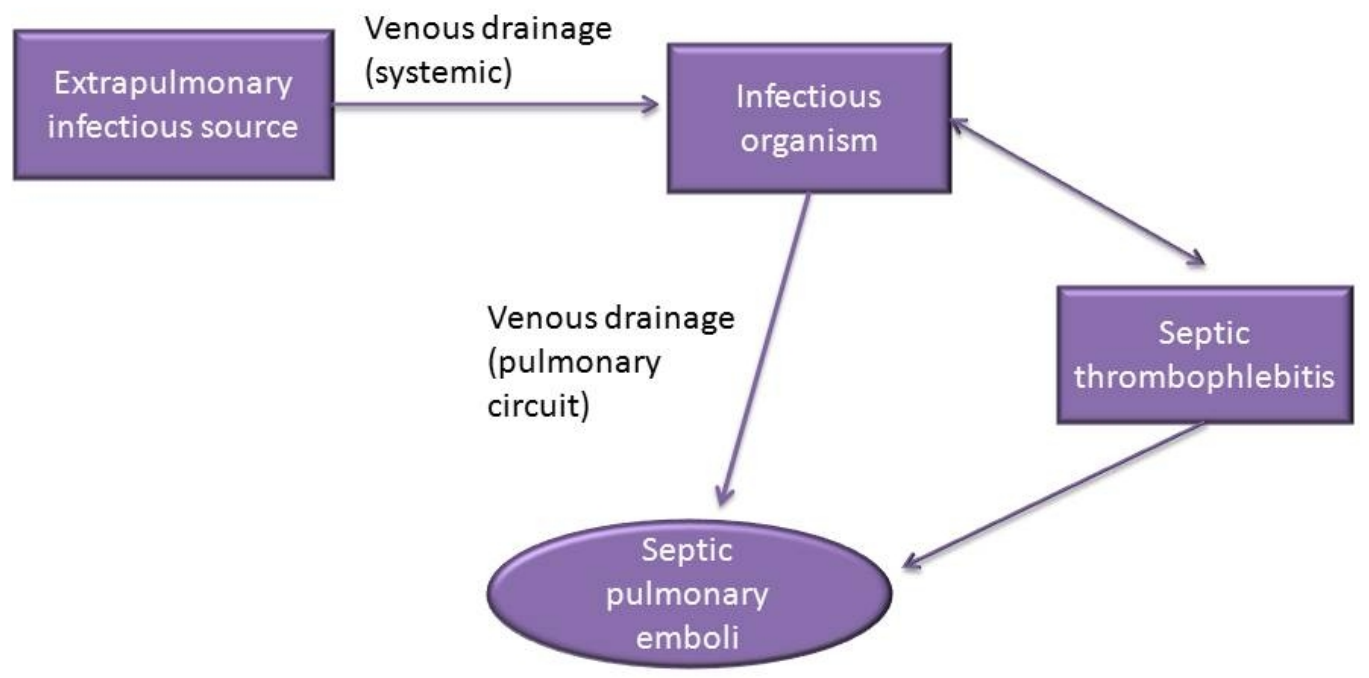

Fig. (2). Schematic of pathophysiology of SPE. 
including: the underlying source (Fusobacterium necrophorum and anaerobes in Lemierrie's syndrome [2, 4]; Bacteroides $\mathrm{sp}$ in pelvic thrombophlebitis etc.) [16, 17]; geography (preponderance of Klebsiella in Korea and SE Asia) [17] and the host (non typhoidal Salmonella in HIV/ AIDS) [18]. Empiric antibiotic therapy should be promptly started, initially with glycopeptides and in appropriate settings, the addition of broad spectrum antibiotics. Antibiotics can then be modified in the light of culture results and continued for a minimum of 4-6 weeks, guided by the clinical improvement and by results of follow up cultures, inflammatory markers and imaging studies.

The role of thoracic surgical treatment has diminished in recent years, due in part to early diagnosis with improvements in imaging and changes in antibiotic prescribing practice [19]. Pulmonary procedures including thoracentesis and chest tube placement remain important. Most patients $(63 \%)$ in our series had some procedure performed including the removal of potentially infected devices, surgical drainage of purulent collections and intracardiac valve replacement.

The role of anticoagulation in the treatment of SPE associated with septic thrombophlebitis remains controversial and these agents should not be initiated until acute infective endocarditis has been excluded. This is largely because of concerns for the possibility of bleeding, particularly in the setting of cerebral embolism [20]. Data to guide the use of anticoagulants and antiplatelet therapies in the presence of infective endocarditis remains limited. Thus, recommendations are based largely on observational studies [21] and expert opinion [22]. Given that there are competing risks of thromboembolism and bleeding, therapy should be individualized in every patient with IE. Recent reviews have emphasized the safety of IV heparin in the treatment of septic thrombophlebitis [23, 24].

The treatment outcomes in cases of SPE depend on the acuity of the illness on initial presentation. Thirteen $(32 \%)$ patients in this series presented acutely ill requiring ICU admission; 8 (20\%) did not survive the hospitalization either due to refractory shock and resultant multi system organ failure or due to grave pulmonary complications including hemorrhage and hemoptysis. Such poor outcome may be related to the late presentation or the presence of severe underlying disease (end stage renal disease, diabetes and immunosuppression) in our patient population.

As evidenced by this relatively large case series, SPE has protean manifestations ranging from an insidious illness with fever and respiratory symptoms to acute sepsis. Physicians should be aware of these clinical presentations as well as the importance of the role of extrapulmonary deep seated infectious sources. Chest CT scans often prompt consideration of this diagnosis and provide the earliest and most easily identifiable indication of systemic embolization of infection. In the absence of ECHO cardiographic or other signs of infective endocarditis further evaluation to identify the infectious source is indicated [25]. Early diagnosis of embolic phenomenon to other organ systems like CNS can greatly impact patient outcome [22]. As with all acute infections, and increasingly so in an era of increased antibiotic resistance, it is essential to obtain prompt blood and other source cultures and to initiate empiric antibiotic therapy, generally directed against staphylococcus aureus. Prolonged antibiotic therapy and procedures aimed at amelioration of the infective source are invariably required.

Limitations of this study include its retrospective nature, and the selection bias involved in a single center study. However, our patient population is likely to be more representative of the larger US community population than previous reports from highly specialized centers which may also explain, in part, the differences in the microbiology and outcome characteristics. Another limitation of the study pertains to the fact that transesophageal echocardiogram was not performed in all patients which limits a complete evaluation of endocarditis.

In conclusion, the epidemiology of SPE has broadened over time with an increase in identified extrapulmonary and non-cardiac sources related to contiguous infections. A clearer understanding of the etiology of SPE will help facilitate the diagnosis and management of this potentially fatal condition.

\section{CONFLICT OF INTEREST}

The authors confirm that this article content has no conflict of interest.

\section{ACKNOWLEDGEMENTS}

Declared none.

\section{REFERENCES}

[1] MacMillan JC, Milstein SH, Samson PC. Clinical spectrum of septic pulmonary embolism and infarction. J Thorac Cardiovasc Surg 1978; 75(5): 670-9.

[2] Goldenberg NA, Knapp-Clevenger R, Hays T, Manco-Johnson MJ. Lemierre's and Lemierre's-like syndromes in children: survival and thromboembolic outcomes. Pediatrics 2005; 116(4): e543-8.

[3] Gorenstein A, Gross E, Houri S, Gewirts G, Katz S. The pivotal role of deep vein thrombophlebitis in the development of acute disseminated staphylococcal disease in children. Pediatrics 2000; 106(6): E87.

[4] Cook RJ, Ashton RW, Aughenbaugh GL, Ryu JH. Septic pulmonary embolism: presenting features and clinical course of 14 patients. Chest 2005; 128(1): 162-6.

[5] Brenes JA, Goswami U, Williams DN. The association of septic thrombophlebitis with septic pulmonary embolism in adults. Open Respir Med J 2012; 6: 14-9.

[6] Li JS, Sexton DJ, Mick N, et al. Proposed modifications to the Duke criteria for the diagnosis of infective endocarditis. Clin Infect Dis 2000; 30(4): 633-8.

[7] Naber CK. Staphylococcus aureus bacteremia: epidemiology, pathophysiology, and management strategies. Clin Infect Dis 2009; 48(Suppl 4): S231-7.

[8] Boyle-Vavra S, Daum RS. Community-acquired methicillinresistant Staphylococcus aureus: the role of Panton-Valentine leukocidin. Laboratory investigation 2006; 87(1): 3-9.

[9] Holm K, Frick IM, Bjorck L, Rasmussen M. Activation of the contact system at the surface of Fusobacterium necrophorum represents a possible virulence mechanism in Lemierre's syndrome. Infect Immun 2011; 79(8): 3284-90.

[10] Gonzalez BE, Teruya J, Mahoney DH, et al. Venous thrombosis associated with staphylococcal osteomyelitis in children. Pediatrics 2006; 117(5): 1673-9.

[11] Huang RM, Naidich DP, Lubat E, Schinella R, Garay SM, McCauley DI. Septic pulmonary emboli: CT-radiographic correlation. AJR Am J Roentgenol 1989; 153(1): 41-5.

[12] Iwasaki Y, Nagata K, Nakanishi M, et al. Spiral CT findings in septic pulmonary emboli. Eur J Radiol 2001; 37(3): 190-4. 
[13] Gadkowski LB, Stout JE. Cavitary pulmonary disease. Clin Microbiol Rev 2008; 21(2): 305-33.

[14] Romano L, Spanu T, Calista F, et al. Tsukamurella tyrosinosolvens and Rhizobium radiobacter sepsis presenting with septic pulmonary emboli. Clin Microbiol Infect 2011; 17(7): 1049-52.

[15] Grace CJ, Lieberman J, Pierce K, Littenberg B. Usefulness of blood culture for hospitalized patients who are receiving antibiotic therapy. Clin Infect Dis 2001; 32(11): 1651-5.

[16] Brown CE, Stettler RW, Twickler D, Cunningham FG. Puerperal septic pelvic thrombophlebitis: incidence and response to heparin therapy. Am J Obstet Gynecol United States 1999; 181(1): 143-8.

[17] Lee SJ, Cha SI, Kim CH, et al. Septic pulmonary embolism in Korea: Microbiology, clinicoradiologic features, and treatment outcome. J Infect England 2007; 54(3): 230-4.

[18] Gordon MA, Banda HT, Gondwe M, et al. Non-typhoidal salmonella bacteraemia among HIV-infected Malawian adults: high mortality and frequent recrudescence. AIDS 2002; 16(12): 163341.

[19] Stawicki SP, Firstenberg MS, Lyaker MR, et al. Septic embolism in the intensive care unit. Int J Crit Illn Inj Sci 2013; 3(1): 58.
[20] Hart RG, Kagan-Hallet K, Joerns SE. Mechanisms of intracranial hemorrhage in infective endocarditis. Stroke 1987; 18(6): 1048-56.

[21] Rasmussen RV, Snygg-Martin U, Olaison L, et al. Major cerebral events in Staphylococcus aureus infective endocarditis: is anticoagulant therapy safe? Cardiology 2009; 114(4): 284-91.

[22] Nishimura RA, Otto CM, Bonow RO, et al. 2014 AHA/ACC guideline for the management of patients with valvular heart disease: a report of the American College of Cardiology/American Heart Association Task Force on Practice Guidelines. J Am Coll Cardiol 2014; 63(22): e57-185.

[23] Ang AK, Brown OW. Septic deep vein thrombosis. J Vas Surg 1986; 4(6): 563-6

[24] Falagas ME, Vardakas KZ, Athanasiou S. Intravenous heparin in combination with antibiotics for the treatment of deep vein septic thrombophlebitis: a systematic review. Eur J Pharmacol 2007; 557(2): 93-8.

[25] Vos FJ, Kullberg BJ, Sturm PD, et al. Metastatic infectious disease and clinical outcome in Staphylococcus aureus and Streptococcus species bacteremia. Medicine (Baltimore) 2012; 91(2): 86-94

(C) Goswami et al.; Licensee Bentham Open.

This is an open access article licensed under the terms of the Creative Commons Attribution Non-Commercial License (http://creativecommons.org/licenses/by-nc/3.0/) which permits unrestricted, non-commercial use, distribution and reproduction in any medium, provided the work is properly cited. 